\title{
Special Issue on Advances on Physical Agents 2016
}

\author{
A. Bandera
}

\begin{abstract}
The Workshop on Physical Agents is a forum for information and experience exchange in different areas regarding the concept of embodied agents, especially applied to the control and coordination of autonomous systems: robots, mobile robots, domotics, agents, industrial applications or complex systems. This special issue brings together a selection of revised and extended papers that were first presented at the XVII Workshop on Physical Agents (WAF'2016), which was held on June 16-17, 2016 at the School of Telecommunication Engineering and Information Technology of the University of Málaga (Spain).
\end{abstract}

Index Terms-WAF, Physical Agents

\section{EDITORIAL}

Technology change is continuous. And, although it is obvious that the current significance of artificial intelligence and its physical embodiment within agents was unimaginable only a few years ago, it is expected that new advances on driverless cars, enhanced elder care or robotics technology will draw a -we hope- better future. Unsupervised online annotation of a video stream, real-time control, therapeutic robotics for the elderly, cognitive software architectures... This special issue collects some of the topics that were discussed within the forum provided by the annual WAF conference. During two days on june 2016, more than 30 researchers met at Málaga for exchanging new ideas and strengthening the always necessary human-to-human relationships. The result was 20 contributions and a nice invited talk from the professore Giulio Sandini, currently on the Instituto Italiano di Tecnologia, and one of the padri fondatori of the active vision. But this tangible result was correctly complemented with a good serving of pleasant conversation (and some cold beer with tapa!). The final cocktail was really nice.

Before providing a brief summary of the papers on this issue, I would like to show the gratitude to all the organizers (Joaquín Ballesteros, Juan P. Bandera, Rebeca Marfil, Adrián Romero-Garcés and Luis V. Calderita) and attendants to the workshop. Of course I particularly wish to extend these thanks to everyone who contributed to this special issue (authors and reviewers). And a special hug to Miguel A. Cazorla and Vicente Matellán for their patient and continuous advice and help.

We look forward to seeing you at the new edition of the WAF in 2017.

\section{A. Special issue overview}

The first paper on the issue proposes an approach to create 3D semantic maps from the metric information provided by a RGB-D sensor. Over the metric representation from the

A. Bandera is with the ISIS group, University of Málaga (Spain). E-mail: ajbandera@uma.es sensor, Félix Escalona, Ángel Rodríguez, Francisco GómezDonoso, Jesús Martínez-Gómez and Miguel Cazorla propose to integrate semantic labels that allow to determine the most probable location of objects. When a frame is captured, the RGB-D information is obtained and mapped to a point cloud. Then, the system registers it to a global coordinate frame. Simultaneously, the approach extracts the probability of being an object using a Convolutional Neural Network (CNN). Under certain conditions, the point clouds associated to the new detection and the one that hold the $3 \mathrm{~d}$ object are merged.

In the second paper, Pablo Bernal-Polo and Humberto Martínez-Barberá present an algorithm for orientation estimation based on extended Kalman filter, quaternions and charts. For filter updating, the proposed approach uses measurements from an Inertial Measurement Unit (IMU). The authors have successfully used basic concepts of manifold theory for estimating orientations using quaternions as descriptors. A similar algorithm to the known as Multiplicative Extended Kalman Filter naturally arises in applying these concepts without having to redefine any aspect of the Extended Kalman Filter.

The work by Eva Mogena, Pedro Núñez and José L. González deals with the therapeutic use of robotics. Specifically, a new system for supervising rehabilitation therapies using autonomous robots for elderly people is presented. The therapy in this work is a modified version of the classical Simon Says game, where a robot executes a list of motions and gestures that the patient has to repeat. From the technical point-of-view, the success of this therapy is based on the correct detection and classification of the human gestures. The algorithm analyses sequences of images acquired by a low cost RGB-D sensor. In addition, this paper describes the human-robot interaction performed within the Simon Says game execution.

The fourth paper presents the redesign and the most relevant implementation issues of the Active Grammar-based Modeling architecture (AGM), as well as the latest developments thereof. The choice of using a software architecture for robotics affects the whole development process. The AGM from Luis J. Manso, Luis V. Calderita and Pablo Bustos is a flexible, modular proposal, which has been designed with computation distribution and scalability in mind. In addition to a continuous refactoring of the API library and planner, the most relevant improvements are an enhanced mission specification syntax, support for representations combining symbolic and metric properties, redesigned communication patterns, and extended middleware support.

Finally, the paper by Julián Cristiano, Doménec Puig and Miguel A. Garía presents an efficient closed-loop locomotion control system for biped robots that operates in the joint space. The robots joints are directly driven through control signals generated by a central pattern generator (CPG) network. A 
genetic algorithm is applied in order to find out an optimal combination of internal parameters of the CPG given a desired walking speed in straight line. Feedback signals generated by the robots inertial and force sensors are directly fed into the CPG in order to automatically adjust the locomotion pattern over uneven terrain and to deal with external perturbations in real time. Omnidirectional motion is achieved by controlling the pelvis motion. The performance of the proposed control system has been assessed through simulation experiments on a NAO humanoid robot. 